\title{
Retinotopic Mapping Requires Focal Adhesion Kinase-Mediated Regulation of Growth Cone Adhesion
}

\author{
Stephanie Woo, ${ }^{1}$ Daniel J. Rowan, ${ }^{2}$ and Timothy M. Gomez ${ }^{2}$ \\ ${ }^{1}$ Department of Biochemistry and Biophysics, University of California, San Francisco, California 94158, and ${ }^{2}$ Department of Anatomy and Program in \\ Cellular and Molecular Biology, University of Wisconsin, Madison, Wisconsin 53706
}

\begin{abstract}
Adhesion controls growth cone motility, yet the effects of axon guidance cues on adhesion site dynamics are poorly understood. Here we show that ephrin-A1 reduces retinal ganglion cell (RGC) axon outgrowth by stabilizing existing adhesions and inhibiting new adhesion assembly. Ephrin-A1 activates focal adhesion kinase (FAK) in an integrin- and Src-dependent manner and the effects of ephrin-A1 on growth cone motility require FAK activation. We also find that FAK is expressed in a high temporal to low nasal gradient in RGCs, similar to EphA receptors, and that balanced FAK activation is necessary for optimal axon outgrowth. Last, we find that FAK is required for proper topographic positioning of retinal axons along the anterior-posterior axis of the optic tectum in both Xenopus and zebrafish, a guidance decision mediated in part by A-type ephrins. Together, our data suggest that ephrin-A1 controls growth cone advance by modulating adhesive point contacts through FAK activation and that graded FAK signaling is an important component of ephrin-Amediated retinotopic mapping.
\end{abstract}

\section{Introduction}

Axon pathfinding is a highly coordinated process involving leading-edge membrane protrusion followed by adhesion of new protrusions to the extracellular matrix (ECM) and turnover of old adhesions. While several studies have focused on the mechanisms controlling membrane protrusion, regulation of growth cone adhesion remains poorly understood. Adhesion to ECM proteins occurs through clustered integrin receptors (Hynes, 2002), which link to the actin cytoskeleton through a large complex of adaptor and signaling proteins (Geiger et al., 2001; Frame et al., 2002). Growth cones adhesions are referred to as point contacts (Gomez et al., 1996; Renaudin et al., 1999). Point contacts form near the leading edge of lamellipodial protrusions or within filopodial shafts and remain stable as the growth cone migrates forward (Woo and Gomez, 2006), but eventually disassemble to promote forward advance. The process of point contact formation, stabilization, and turnover is tightly coupled to membrane protrusion, and disruption of point contact dynamics impairs growth cone motility (Varnum-Finney and Reichardt, 1994; Woo and Gomez, 2006).

Focal adhesion kinase (FAK) is a protein tyrosine kinase that regulates axon outgrowth and growth cone turning in culture, as well as axon guidance, branching, and synaptogenesis in vivo ( $\mathrm{Li}$ et al., 2004; Ren et al., 2004; Rico et al., 2004; Robles and Gomez,

Received Aug. 17, 2009; revised Sept. 16, 2009; accepted Sept. 30, 2009

This work was supported by National Institutes of Health Grant NS41564 and a Dana Foundation grant to T.M.G. We thank T. Xiao and H. Baier (University of California, San Francisco) for providing the pou4f3:Gal4 fish and for helpful advice. We also thank K. Kalil, M. Halloran, and members of the Gomez laboratory for comments on this manuscript and $C$. Trevor for help with statistical analysis.

Correspondence should be addressed to Timothy M. Gomez, University of Wisconsin School of Public Health, 257 Bardeen Laboratories, 1300 University Avenue, Madison, W1 53706. E-mail: tmgomez@wisc.edu.

DOI:10.1523/JNEUROSCI.4028-09.2009

Copyright $\odot 2009$ Society for Neuroscience ～0270-6474/09/2913981-11\$15.00/0
2006). In fibroblasts, FAK is one of the first proteins to localize to focal adhesions and increased FAK phosphorylation correlates with focal adhesion formation, which suggests that FAK plays a role in either adhesion assembly or maturation (Kirchner et al., 2003). In Xenopus spinal neuron growth cones, loss of FAK activity is associated with decreased paxillin immunostaining, suggesting that FAK is similarly required for formation of neuronal point contacts (Robles and Gomez, 2006). However, $\mathrm{fak}^{-/-} \mathrm{fi}-$ broblasts contain enlarged focal adhesions with impaired rates of disassembly, suggesting that FAK is also required for adhesion turnover (Webb et al., 2004).

Ephrins are repulsive cues that function during axon guidance, as well as many other developmental processes (Pasquale, 2005). Ephrin ligands bind Eph receptors and activate RhoA signaling through the guanine nucleotide exchange factor called ephexin (Shamah et al., 2001). However, other signals appear to mediate the effects of ephrin on growing axons. For example, Src family kinases interact with EphA receptors, phosphorylate ephexin (Sahin et al., 2005), and are required for retinal axons to respond to ephrin-A (Knöll and Drescher, 2004). In addition, FAK has been shown to act downstream of A-type ephrins, but these reports are contradictory. For example, ephrin-A1 was shown to increase FAK phosphorylation and adhesion of cultured fibroblasts (Carter et al., 2002), whereas other studies have shown that ephrin-A promotes FAK dephosphorylation and decreased cell adhesion (Miao et al., 2000; Bourgin et al., 2007).

In this study, we report that a low dose of ephrin-A1 reduces membrane protrusion and stabilizes growth cone point contacts of Xenopus retinal neurons. The effects of ephrin-Al depend on FAK and Src activation in growth cones. Interestingly, ephrin-A1 increases FAK phosphorylation in temporal retinal axons to a level higher than that in nasal axons, which correlates with the differential response of these axons to ephrin-A1. Moreover, sim- 
ilar to EphA receptors, FAK expression is higher in temporal versus nasal retinal ganglion cells (RGCs). Finally, we show that inhibition of FAK function in vivo leads to retinotopic errors in developing Xenopus and zebrafish. Together, our results show that modulating adhesion and adhesion-associated signaling pathways is an important component of regulating axon outgrowth in vitro and axon guidance in vivo.

\section{Materials and Methods}

Expression constructs and embryo injection. Expression constructs were subcloned into the Xenopus-optimized pCS + vector (Dave Turner, University of Michigan, Ann Arbor, MI). cDNA for chicken paxillin-GFP was provided by A. F. Horwitz (University of Virginia, Charlottesville, VA). GFP-FRNK was provided by P. Keely (University of Wisconsin, Madison, WI). The xFAK morpholino was designed and purchased from Gene Tools as previously described (Robles and Gomez, 2006). Xenopus laevis embryos were obtained as described previously (Gómez et al., 2003) and staged according to Nieuwkoop and Faber (1994). For direct expression experiments, two dorsal blastomeres of eight-cell-stage embryos were injected with $0.5-1 \mathrm{ng}$ of in vitro-transcribed, capped mRNA (mMessage Machine, Ambion) or 100 pg of DNA for paxillin-GFP. For morpholino experiments, we injected $2-4 \mathrm{nl}$ of $1 \mathrm{~mm}$ FAK morpholino combined with $10 \mathrm{mg} \mathrm{ml}^{-1}$ Alexa-488-conjugated dextran (Invitrogen) or paxillin-GFP DNA. For in vivo experiments, $0.5-1 \mathrm{ng}$ of control GFP mRNA or GFP-FRNK mRNA was injected into the RD1 blastomere of eight-cell-stage embryos to restrict expression to the right eye and forebrain (Hirose and Jacobson, 1979). Alternatively, for delayed expression by heat shock, transgenesis was performed as described previously (Ogino et al., 2006). Briefly, the zebrafish $h s p 70$ promoter (Halloran et al., 2000) and GFP-FRNK were subcloned into the pBSII-IsceI vector (Thermes et al., 2002) and flanked by I-SceI meganuclease recognition sites. Twenty-five nanograms of DNA was incubated with 20 units I-SceI enzyme (New England Biolabs) for $40 \mathrm{~min}$ at $37^{\circ} \mathrm{C}$. Two nanoliters of the reaction mixture was injected into one- or two-cell-stage embryos. Under these conditions, expression of the transgene is restricted to either the left or right half in $\sim 20 \%$ of embryos injected (Ogino et al., 2006); analysis was restricted to embryos with only one eye labeled after induction of transgene expression. To induce expression, stage 28 embryos were heat shocked at $37^{\circ} \mathrm{C}$ for $1 \mathrm{~h}$. Zebrafish $h s p 70$ was provided by $\mathrm{M}$. Halloran (University of Wisconsin, Madison, WI). pBSII-IsceI was provided by M. Sheets (University of Wisconsin, Madison, WI).

Retinal cell culture and reagents. For retinal cultures, whole eye primordia (see Figs. 1-4) were dissected from stage 28-29 embryos and explants were cultured in $60 \%$ L15 medium (Sigma) as described previously (Woo and Gomez, 2006). Explants were cultured on loose glass coverslips precoated with $50-100 \mu \mathrm{g} \mathrm{ml}^{-1}$ poly-D-lysine (PDL, Sigma) followed by 10 $\mu \mathrm{g} \mathrm{ml}^{-1}$ laminin (LN) (Sigma). For nasal and temporal RGC cultures, the extreme $1 / 8$ to $1 / 10$ of the nasal and temporal retina was dissected from eyes (see Fig. 5a). Cultures were imaged or fixed 12-24 h after plating. Recombinant mouse ephrin-A1-Fc was purchased from R\&D Systems. Human IgG, Fc fragment was purchased from Jackson ImmunoResearch Laboratories. Herbimycin A (HA) was purchased from Biomol. Function-blocking $\beta 1$-integrin antibody was provided by K. Yamada (AB2999; National Institute of Dental and Craniofacial Research, National Institutes of Health, Bethesda, MD).

Immunocytochemistry, immunohistochemistry, and immunoblotting. For immunocytochemistry (ICC), cultures were fixed in $4 \%$ paraformaldehyde $/ 4 \%$ sucrose in calcium- and magnesium-free PBS (CMF-PBS) followed by permeabilization in $0.1 \%$ Triton X-100 in CMF-PBS and blocked in 1\% fish gelatin (Sigma) in CMF-PBS for $1 \mathrm{~h}$ at room temperature. Primary antibodies were used at the following dilutions in blocking solution: 1:500 all pY-FAK antibodies (Biosource), 1:500 total FAK (Abcam and clone 4.47, Millipore), 1:200 Fc (Jackson ImmunoResearch Laboratories). Alexa-Fluor-conjugated secondary antibodies were purchased from Invitrogen and used at 1:250 in blocking solution. Included with secondary antibodies was Alexa-546 phalloidin (1:50-1:100; Invitrogen) to label filamentous actin (F-actin) and 5-(4,6-dichlorotriazinyl) aminofluorescein (1:1000; DTAF; Invitrogen) or Alexa Fluor 647 carboxylic acid, suc- cinimidyl ester (1:1000; SE-647, Invitrogen) to label total protein content. For cryostat sectioning, staged embryos were fixed overnight at $4^{\circ} \mathrm{C}$ in $4 \%$ paraformaldehyde $/ 4 \%$ sucrose. After extensive washing in CMF-PBS, embryos were sunk in $30 \%$ sucrose and then frozen on dry ice in Tissue-Tek O.C.T. compound (Sakura Finetechnical) and $14 \mu \mathrm{m}$ horizontal sections were made using a cryostat. Sections were refixed for 10 min in $4 \%$ paraformaldehyde $/ 4 \%$ sucrose and then washed extensively in rinse solution $(0.1 \%$ Triton X-100/0.1\% fish gelatin in CMF-PBS). After $1 \mathrm{~h}$ in blocking solution $(0.1 \%$ Triton $\mathrm{X}-100 / 1.0 \%$ fish gelatin in CMFPBS), primary antibodies were applied overnight at $4^{\circ} \mathrm{C}$ in blocking solution. Anti-Islet-1 (University of Iowa Developmental Studies Hybridoma Bank) was used at 1:100 and other primary antibodies were used as described previously. Immunoblotting was performed as described previously (Robles et al., 2003) using lysates from nasal or temporal halves of stage 28 eye primordia. For immunoblotting, FAK (Abcam) and $\beta$-tubulin (Sigma) antibodies were used at 1:1000. Horseradish peroxidase-conjugated secondary antibodies were purchased from Santa Cruz Biotechnology and used at 1:5000-1:10,000.

Image acquisition and analysis. For both live and fixed samples, highmagnification images were acquired using either a $60 \times / 1.45$ NA objective lens on an Olympus Fluoview 500 laser-scanning confocal system mounted on an AX-70 upright microscope or a $100 \times / 1.5$ NA objective lens on a Nikon total internal reflection microscope. On the confocal, samples were imaged at 2-2.5× zoom (pixel size $=165-200 \mathrm{~nm}$ ). Lowmagnification phase-contrast images were acquired using a $20 \times$ objective on Nikon microscope equipped with an $x-y$ motorized stage for multipositional imaging. Live explant cultures were sealed in enclosed perfusion chambers as described previously (Gómez et al., 2003) to allow rapid exchange of solutions. Images were analyzed using either MetaMorph (Universal Imaging) or ImageJ software (W. Rasband, National Institutes of Health, Bethesda, MD). Point contacts were identified as discrete areas containing paxillin-GFP that were at least two times brighter than the surrounding background and remained fixed in place for a minimum of $30 \mathrm{~s}$ (Woo and Gomez, 2006). Kymographs were created using MetaMorph or ImageJ along lines 1 pixel in width. Protrusions were measured if the leading-edge membrane exhibited a positive slope with a minimum rise and run of $1.5 \mu \mathrm{m}$ and $30 \mathrm{~s}$, respectively. Quantifications of pY-FAK and total protein were made by first selecting the perimeter of growth cones from thresholded total protein or F-actinlabeled images based on intensity to exclude background using MetaMorph or ImageJ. These user-defined regions were then used to measure the total or average pixel intensity of pY-FAK and total protein fluorescence within nonthresholded growth cones. For display purposes, some images were pseudocolored in ImageJ. To quantify FAK labeling across the RGC labeling retina, the average intensity of FAK and Islet-1 labeling within a 10-pixel-wide line through the RGC layer (identified by Islet labeling) was measured using MetaMorph. Intensities along each line were binned into $5 \%$ intervals to normalize for differences in line length between individual retinas and averaged. The intensity of FAK labeling within terminal quadrants of the RGC layer was quantified by first selecting the RGC layer by thresholding the Islet-1 fluorescence channel. The nasal and temporal quadrants of the RGC layer were then selected within the thresholded region and the average fluorescence intensity of FAK labeling was measured. For most figures, images were processed in Adobe Photoshop (Adobe Systems) as follows: brightness levels adjusted, unsharp mask routine to improve edge detection, converted to 8 -bit depth, and cropped. Statistical significance was determined using Mann-Whitney or Student's $t$ test and variance reported as \pm SEM from at least two independent experiments using InStat or Prism software (GraphPad Software).

In vivo labeling of retinotectal pathway. Embryos were raised to stages 45-47 in $0.1 \mathrm{~mm}$ phenylthiourea in $0.1 \times$ modified Ringer's solution to inhibit pigment synthesis and facilitate dye injection. Embryos were fixed overnight at $4^{\circ} \mathrm{C}$ in $4 \%$ paraformaldehyde $/ 4 \%$ sucrose in CMF-PBS. To label nasal and temporal retinal projections, a $1 \%$ solution of $\mathrm{DiD}\left(1,1^{\prime}\right.$ dioctadecyl-3,3,3', $3^{\prime}$-tetramethylindodicarbocyanine perchlorate) or DiI (1,1' -dioctadecyl-3,3,3',3'-tetramethylindocarbocyanine perchlorate; Invitrogen) in dimethylformamide was injected in the nasal (DiD) or temporal (DiI) half of the right eye. The dyes were allowed to transport 

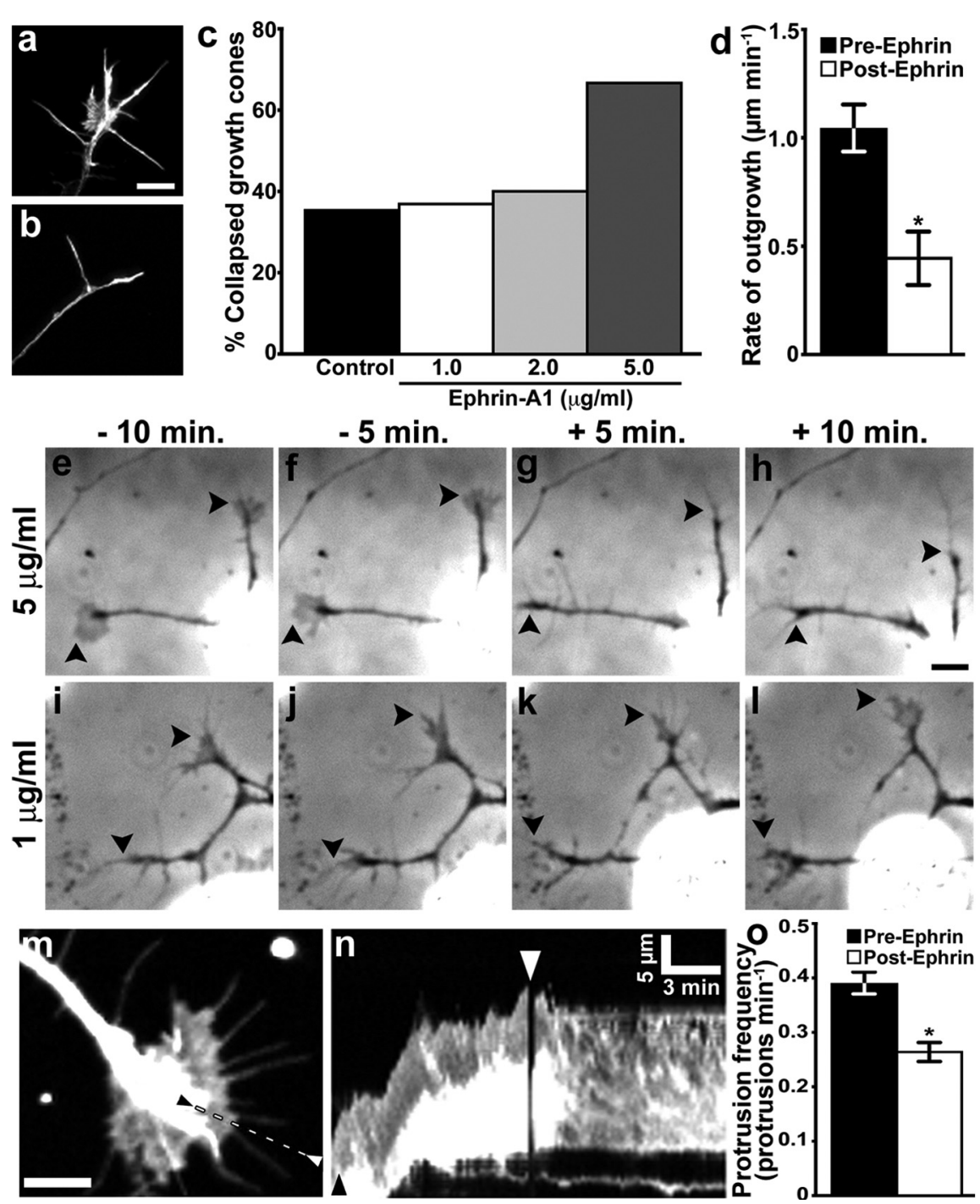

Figure 1. Low-dose ephrin-A1 reduces growth cone motility without inducing collapse. $\boldsymbol{a}, \boldsymbol{b}, \mathrm{RGC}$ growth cones cultured from total retina treated for 10 min with control medium $(\boldsymbol{a})$ or $5 \mu \mathrm{g} \mathrm{ml}{ }^{-1}$ ephrin-A1 $(\boldsymbol{b})$. Growth cones were stained with fluorescent phalloidin to label F-actin. c, Percentage of collapsed growth cones after a 10 min treatment with $1(n=40), 2$ ( $n=38)$, or 5 ( $n=$ 36) $\mu \mathrm{g} \mathrm{ml}^{-1}$ ephrin-A1 or control medium $(n=82)$. $\boldsymbol{d}$, Average rate of neurite outgrowth before and after the addition of $1 \mu \mathrm{g}$ $\mathrm{ml}^{-1}$ ephrin-A1 ( $\left.n=21\right) \cdot \boldsymbol{e}-\boldsymbol{l}, \mathrm{RGC}$ growth cones (arrowheads) at the indicated times before and after treatment with $5(\boldsymbol{e}-\boldsymbol{h})$ or $1(\boldsymbol{i}-\boldsymbol{I}) \mu \mathrm{g} \mathrm{ml}^{-1}$ ephrin-A1. Growth cones collapse and axons retract in response to $5 \mu \mathrm{g} \mathrm{ml}^{-1}$ but not $1 \mu \mathrm{g} \mathrm{ml}^{-1}$ ephrin-A1. $\boldsymbol{m}$, $\boldsymbol{n}$, RGC growth cones expressing GFP were imaged for $10 \mathrm{~min}$ before and after the addition of $1 \mu \mathrm{g} \mathrm{ml}^{-1}$ ephrin-A1. The kymograph in $\boldsymbol{n}$ was generated along the dashed line between arrowheads shown in $\boldsymbol{m}$. Note that after ephrin addition (white arrowhead), the leading edge ceases to advance forward and there are few membrane protrusions. The black arrowhead indicates time point of the still image in $\boldsymbol{m}$. $\boldsymbol{o}$, Ephrin-A1 $\left(1 \mu \mathrm{g} \mathrm{ml}^{-1}\right)$ significantly reduces the frequency of leading-edge membrane protrusion. ${ }^{*} p<0.05, n=28$. Scale bars: $\boldsymbol{a}-\boldsymbol{I}, 10 \mu \mathrm{m} ; \boldsymbol{m}, 5 \mu \mathrm{m}$; calibration as indicated in $\boldsymbol{n}$.

overnight at room temperature. To visualize projections, embryos were pinned onto Sylgard-bottom dishes (Corning) and the left eye and surrounding skin were removed. Confocal $z$-stacks were acquired using a $20 \times / 0.5$ NA objective lens at $4 \mu \mathrm{m}$ steps and displayed as maximumintensity projections. Image analysis was performed using Image or $\mathrm{Vo}$ locity (Improvision) software. Three methods were used to analyze the separation of the nasal and temporal topographic maps. The Pearson's correlation coefficient measures the degree of overlap between two fluorescent signals, with perfect overlap assigned a value of one. For this, $z$-stacks of DiI and DiD fluorescence images were thresholded based on intensity to highlight labeled axons within the tectum using Volocity software. For the second measurement, the $x-y$ position of the center of mass (CM) of thresholded DiI and DiD maximum projected 2D images were determined using ImageJ software. The distance separating each $\mathrm{CM}$ was then measured. A third measurement was the distance to the midbrain-hindbrain boundary, which is a consistently identifiable landmark. Here the distance of the most posterior DiI- and DiDlabeled axons to the midbrain-hindbrain boundary was measured to determine the relative position of each arbor.

Zebrafish experiments. Twenty to twenty-five picograms of plasmid DNA encoding either UAS:GFP or UAS:GFP-FRNK was injected into $\mathrm{Tg}$ (pou4f3:GAL4) ${ }^{\text {s311t }}$ transgenic embryos at the one-cell stage. At 5 days postfertilization, embryos were fixed in $4 \%$ paraformaldehyde in PBS overnight at $4^{\circ} \mathrm{C}$. To label retinotectal projections, a $1 \%$ solution of $\mathrm{DiD}$ or $\mathrm{DiI}$ was injected in the nasal or temporal half of the right eye. Dyes were allowed to transport overnight at room temperature. Embryos were embedded in $1 \%$ agarose in PBS for imaging. Confocal $z$-stacks were acquired on a Zeiss LSM5 Pascal confocal microscope, using a $40 \times 1.75 \mathrm{NA}$ objective lens at $2 \mu \mathrm{m}$ steps. The $x-y$ position of the CM of thresholded maximum projected 2D images of GFP fluorescence was determined using ImageJ software. The CM of GFP-positive axon arbors was measured to the anterior and posterior boundaries of the tectum identified by DiD and DiI labeling.

\section{Results}

Noncollapsing doses of ephrin-A1 decrease growth cone motility

To determine the effects of ephrin-A1 on point contacts, we first determined the concentration of ephrin-A1 that would cause limited collapse of the growth cone peripheral domain. We treated Xenopus RGC neurons cultured on LN from total retina with 1,2 , or $5 \mu \mathrm{g} \mathrm{ml}^{-1}$ ephrin-A1 or control media for $10 \mathrm{~min}$. To assess the dose-dependent effects on collapse, growth cones were fixed and stained with fluorescent phalloidin to label F-actin (Fig. 1a,b). Growth cones were scored as collapsed if their peripheral lamellipodia was less then $30 \%$ of the total growth cone area and growth cones had fewer than three filopodia, as described previously (Cox et al., 1990). We found that $5 \mu \mathrm{g}$ $\mathrm{ml}^{-1}$ ephrin-A1 resulted in the collapse of $66.7 \%$ of growth cones $(n=36)$, compared with $35.4 \%$ collapsed under control conditions $(n=82)$. However, at 1 and 2 $\mu \mathrm{g} \mathrm{ml}^{-1}$ ephrin-A1, we find that the incidence of growth cone collapse is not significantly greater than what was seen for control media (Fig. 1c). Furthermore, when observed by time-lapse microscopy, $5 \mu \mathrm{g} \mathrm{ml}^{-1}$ ephrin-A1 induced rapid axon retraction (Fig. $1 e-h)$, consistent with previously published reports (Shamah et al., 2001), while axon retraction was infrequent at 1 $\mu \mathrm{g} \mathrm{ml}^{-1}$ ephrin-A1 (Fig. $1 i-l$ ).

Although RGC growth cones do not collapse in response to low-dose ephrin-A1, we find that the rate of neurite outgrowth was significantly reduced by $1 \mu \mathrm{g} \mathrm{ml}^{-1}$ ephrin-A1, from $1.04 \pm$ $0.12 \mu \mathrm{m} \mathrm{min}^{-1}$ to $0.44 \pm 0.12 \mu \mathrm{m} \mathrm{min}^{-1}(n=28, p<0.001)$ (Fig. 1d). Ephrin-A1 appears to inhibit neurite outgrowth by altering membrane protrusion, which we quantified by kymog- 
raphy (Fig. $1 m-o$ ). Reduced protrusion is clear in the kymographs (Fig. 1n), as the slope of the leading edge becomes level upon addition of ephrin-A1. Before ephrin-A1 addition, membrane protrusion occurred at a frequency of $0.39 \pm$ 0.02 protrusions $\min ^{-1}$, which decreased to $0.26 \pm 0.02$ protrusions $\min ^{-1}$ after addition of $1 \mu \mathrm{g} \mathrm{ml}^{-1}$ ephrin-A1 $(n=28$, $p<0.0001$ ) (Fig. 1o). Thus, even in the absence of complete growth cone collapse, a low dose of ephrin-A1 decreases growth cone motility.

\section{Ephrin-A1 decreases point contact turnover}

Previously we showed that the formation and turnover of integrin-dependent adhesion sites called point contacts influences growth cone motility in vitro and axon guidance in vivo (Robles and Gomez, 2006; Woo and Gomez, 2006). Therefore, we reasoned that the inhibitory effects of ephrin-A1 on growth cone motility might also be due to changes in point contact dynamics. To test this possibility, we expressed paxillin-GFP in RGC neurons and cultured total retina on LN. Paxillin is an adaptor protein that localizes to sites of integrin-mediated adhesion (Turner, 2000), and paxillin-GFP has previously been shown to be a reliable marker for growth cone point contacts (Woo and Gomez, 2006). Live paxillin-GFP-expressing growth cones were imaged by confocal or total internal reflection fluorescence microscopy for 10-15 min before and after addition of $1 \mu \mathrm{g} \mathrm{ml} \mathrm{m}^{-1}$ ephrin-A1. Before addition of ephrin-A1, point contacts form at the leading edge of new protrusions, stabilize, and turn over rapidly as growth cones advance forward (Fig. $2 a, b, e, g$ ). Addition of ephrin-A1 has two effects on point contacts. First, there is a decrease in the frequency of new point contact formation. On average, growth cones formed $1.82 \pm 0.28$ point contacts $\min ^{-1}$ before ephrin-A1 addition and this frequency dropped to $1.18 \pm$ 0.22 point contacts $\mathrm{min}^{-1}$ in the presence of ephrin-A1 $(n=14$ growth cones, $p<0.05$ ). Second, pre-existing point contacts and those that form after ephrin treatment become overstabilized and turn over more slowly (Fig. $2 c, d, f, h$ ). In some cases, this was associated with a near-complete arrest of growth cone migration (supplemental Movie 1, available at www.jneurosci.org as supplemental material). In the presence of ephrin-A1, the average point contact lifetime increased from $91.2 \pm 3.7$ to $122.4 \pm 6.5 \mathrm{~s}$ ( $n=14$ growth cones, $p<0.0001$ ). This effect is best illustrated by viewing kymographs through individual point contacts that formed before (Fig. $2 g$ ) and after (Fig. $2 h$ ) ephrin-Al treatment. Thus, ephrin-A1 increases point contact lifetime, but decreases new point contact formation, leading to reduced point contact dynamics overall, which may contribute to decreased growth cone motility.

\section{Ephrin-A1 alters FAK phosphorylation at specific tyrosine residues}

EphA receptors may modulate integrin-dependent adhesion by activating signaling pathways, such as the small GTPases RhoA and Rac1(Wahl et al., 2000; Shamah et al., 2001; Jurney et al. 2002), as well as the protein tyrosine kinases Src and FAK (Miao et al., 2000; Carter et al., 2002; Knöll and Drescher, 2004; Sahin et al., 2005). FAK is particularly intriguing, since it is enriched within point contacts and is required for point contact assembly and growth cone motility (Gomez et al., 1996; Renaudin et al., 1999; Robles and Gomez, 2006). However, in non-neuronal cells, the role of FAK downstream of EphA receptors is contradictory (Miao et al., 2000; Carter et al., 2002), so it is unclear what effects, if any, ephrin-A1 will have on FAK activity within RGC growth cones.

FAK activity is modulated by phosphorylation on several functionally distinct tyrosine residues. For example, Y397 is an auto-phosphorylation site involved with the initial activation of FAK and subsequently binds Src (Mitra et al., 2005), which then phosphorylates Y861 to promote cell motility (Lim et al., 2004). On the other hand, phosphorylation of Y407 has been shown to negatively regulate FAK function (Jeon et al., 2007). To determine the effects of ephrin-A1 on FAK activity, RGC growth cones on LN were treated with ephrin-A1 or control media for 5 min and then fixed and processed for ICC using several phosphospecific FAK antibodies (pY-397, pY-407, pY-576, and pY-861) (Fig. 3).

Five minutes of treatment with 1 or $2 \mu \mathrm{g} \mathrm{ml}{ }^{-1}$ ephrin-A1 caused a dose-dependent increase in the average fluorescence intensity of pY-397 FAK within growth cones (Fig. $3 a-d, q$ ). Similar results were observed when the amount of pY-FAK was expressed as a ratio of the pY-FAK immunolabeling relative to total protein staining (pY-FAK/protein) (supplemental Fig. 1, available at www.jneurosci.org as supplemental material) (Schindelholz and 

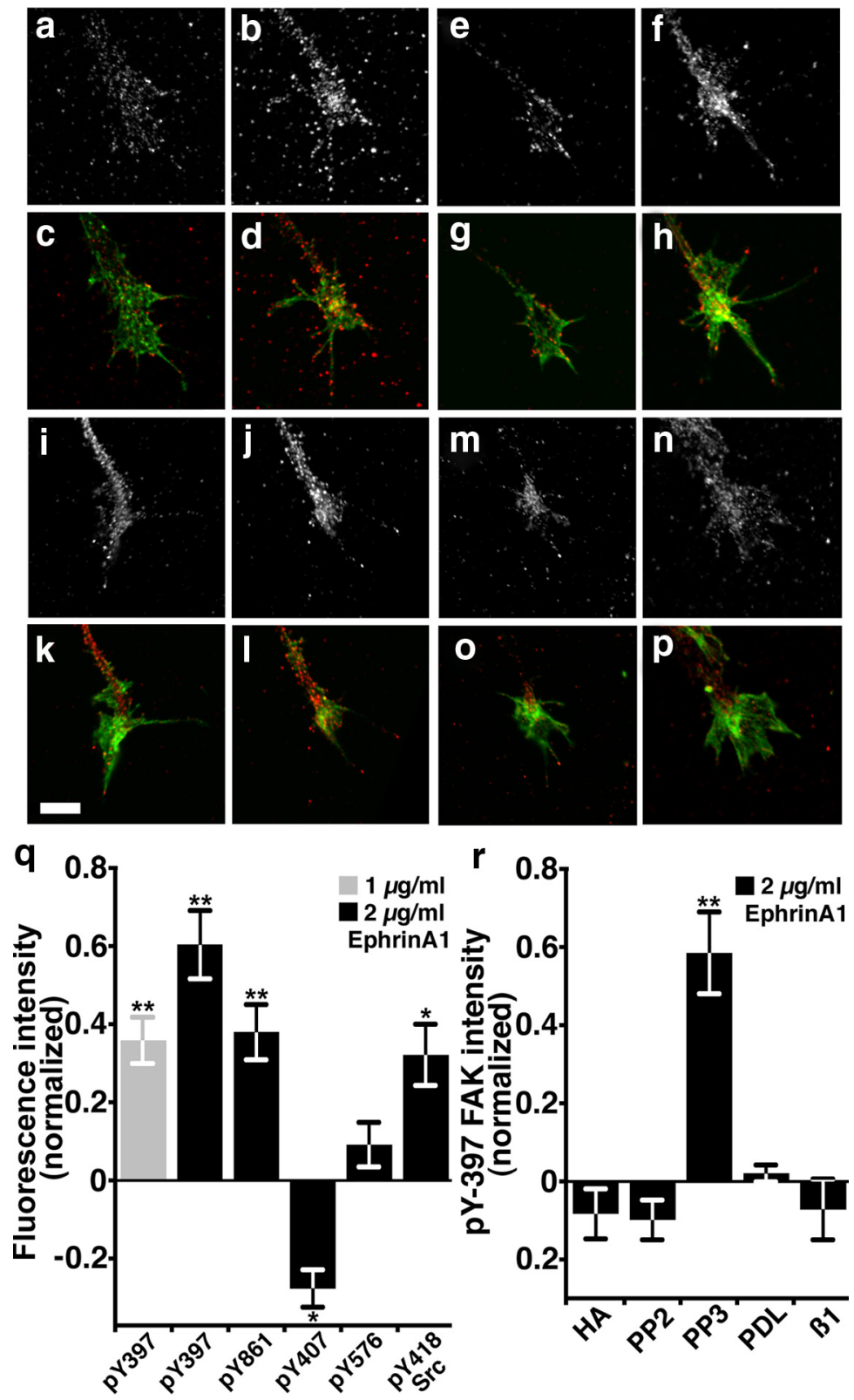

Figure 3. Ephrin-A1 activates FAK and Src signaling. $\boldsymbol{a}-\boldsymbol{d}$, Representative growth cones labeled with an antibody that recognizes pY-397 FAK $(\boldsymbol{a}, \boldsymbol{b})$ together with fluorescent phalloidin to label F-actin $(\boldsymbol{c}, \boldsymbol{d})$. Growth cones treated with $2 \mu \mathrm{g} \mathrm{ml}{ }^{-1}$ ephrin-A1 for $5 \mathrm{~min}(\boldsymbol{b}, \boldsymbol{d})$ appear to have higher pY-397 FAK staining compared with mock-treated control growth cones $(\boldsymbol{a}, \boldsymbol{c})$ $\boldsymbol{e}-\boldsymbol{h}$, Representative control growth cones labeled with an antibody that recognizes pY-861 FAK $(\boldsymbol{e}, \boldsymbol{f})$ together with fluorescent phalloidin to label F-actin $(\boldsymbol{g}, \boldsymbol{h})$. Growth cones treated with $2 \mu \mathrm{g} \mathrm{ml}{ }^{-1}$ ephrin-A1 for $5 \mathrm{~min}(\boldsymbol{f}, \boldsymbol{h})$ appear to have higher pY-861 FAK staining compared with untreated growth cones $(\boldsymbol{e}, \boldsymbol{g})$. $\boldsymbol{i}-\boldsymbol{l}$, Representative growth cones labeled with an antibody that recognizes pY-418 Src $(\boldsymbol{i}, \boldsymbol{j})$ together with fluorescent phalloidin to label F-actin $(\boldsymbol{k}, \boldsymbol{I})$. Growth cones treated for 5 min with $2 \mu \mathrm{g}$ $\mathrm{ml}^{-1}$ ephrin-A1 $(\mathbf{j}, \boldsymbol{I})$ appear to have higher pY-418 Src staining compared with mock-treated control growth cones $(\boldsymbol{i}, \boldsymbol{k}) . \boldsymbol{m}-\boldsymbol{p}$, Representative growth cones cultured on PDL labeled with an antibody that recognizes pY-397 FAK $(\boldsymbol{m}, \boldsymbol{n})$ together with fluorescent phalloidin to label F-actin $(\boldsymbol{o}, \boldsymbol{p})$. Growth cones treated for 5 min with $2 \mu \mathrm{g} \mathrm{ml}{ }^{-1}$ ephrin-A1 on PDL $(\boldsymbol{n}, \boldsymbol{p})$ appear to have no difference pY-397 FAK staining compared with mock-treated control growth cones $(\boldsymbol{m}, \boldsymbol{0})$. $\boldsymbol{q}$, Quantification of pY-FAK and pY-Src labeling in growth cones. The average fluorescence intensity of PY labeling within growth cones was normalized against growth cones treated with control media for each experimental condition. $n \geq 34$ growth cones from two to four cultures for each condition. $r$, Ephrin-induced changes in pY-397 FAK were blocked by pretreating growth cones for 5-10 min with the general tyrosine kinase inhibitor HA (2 $\mu \mathrm{M})$, or with a Src family kinase-specific inhibitor (PP2, $2 \mu \mathrm{m})$, or by culturing neurons on a non-integrin-binding substratum (PDL), or by pretreating neurons on $L N$ with a $\beta 1$-integrin function-blocking antibody $(\beta 1,100$ $\mu \mathrm{g} / \mathrm{ml}$ ). $n \geq 35$ growth cones from two to four cultures for each condition. ${ }^{*} p<0.05 .{ }^{* *} p<0.001$. Scale bar, $5 \mu \mathrm{m}$.

Reber, 1999), suggesting that increased pY-FAK is not due to changes in growth cone morphology. Since $2 \mu \mathrm{g} \mathrm{ml}^{-1}$ ephrin-A1 resulted in a stronger activation of pY-397 FAK (Fig. 3q), this subcollapsing dose of ephrin (Fig. 1c) was used for all subsequent experiments. Interestingly, ephrin-A1 treatment also increased phosphorylation of FAK at Y861 (Fig. 3e-h), but led to a significant decrease in Y407 phosphorylation (Fig. 3q). Y576 FAK phosphorylation was not significantly effected by ephrin treatment. Consistent with previous studies showing the activation of Src by ephrin (Knöll and Drescher, 2004) we find that Y418 Src is also phosphorylated in response to ephrin-A1 (Fig. 3i-l,q). To confirm the specificity of our pY assay, we found that the general tyrosine kinase inhibitor HA and the Src family kinase inhibitor PP2 prevented phosphorylation of Y397 FAK, while PP3 (an inactive analog of PP2) had no effect (Fig. $3 r$; supplemental Fig. $1 F$, available at www.jneurosci.org as supplemental material). Interestingly, ephrin activation of FAK at Y397 required integrin engagement, since both culturing neurons on the non-integrin-binding substratum PDL (Fig. $3 m-p$ ), or pretreating neurons cultured on LN with a $\beta 1$-integrin function-blocking antibody, prevented changes in pY-397 FAK levels (Fig. 3r). Together, these results suggest that ephrin-Al activates FAK in an integrinand Src-dependent manner.

FAK and Src activity are required for ephrin-A1-induced effects on growth cone motility and point contact dynamics

To assess whether activation of FAK is necessary for ephrin-A1 inhibition of outgrowth, we examined growth cones with reduced FAK function. FAK activity was decreased in neurons by two independent means. First, FAK was inhibited by overexpression of an inhibitory splice variant, FAK-related non-kinase (FRNK). This variant of FAK acts as a dominant negative since it contains the C-terminal protein-protein interaction domains but lacks the kinase and FERM domains (Schlaepfer et al., 2004). Second, FAK expression was reduced using an antisense morpholino oligonucleotide directed against Xenopus FAK (FAKmo). The translationblocking morpholino we used has previously been shown to reduce FAK protein levels by $\sim 50 \%$ when introduced at doses that do not interfere with gross embryogenesis (Robles and Gomez, 2006).

Live growth cones expressing GFPFRNK or containing FAKmo plus a fluorescent tracer (Alexa-488 dextran) were imaged for 10-15 min before and after the addition of $1 \mu \mathrm{g} \mathrm{ml}^{-1}$ ephrin-A1. Consistent with previous stud-
ies (Robles and Gomez, 2006), strongly labeled neurons, which presumably have the most severe FAK deficiency, typically exhibited short axons and immobile growth cones (data not shown). 
As our goal is to determine whether FAK also functions to reduce outgrowth in response to ephrin-A1, we selected FAKdeficient growth cones that were motile before ephrin-A1 treatment (rate of outgrowth $>0.3 \mu \mathrm{m} \mathrm{min}{ }^{-1}$ ). Among these FAK-deficient neurons, the average rate of neurite outgrowth $(0.79 \pm 0.08 \mu \mathrm{m}$ $\min ^{-1}, n=42$ ) was slightly less than control neurons $\left(1.04 \pm 0.12 \mu \mathrm{m} \mathrm{min}^{-1}, n=\right.$ 28, $p=0.06$ ) (supplemental Fig. $2 A$, available at www.jneurosci.org as supplemental material), suggesting a partial but not complete loss of FAK activity. However, unlike wild-type neurons, addition of $1 \mu \mathrm{g} \mathrm{ml}^{-1}$ ephrin-A1 had no effect on neurite outgrowth in either GFP-FRNKexpressing or FAKmo neurons (Fig. 4c). Analysis of membrane protrusion by kymography also shows that FAK-deficient growth cones are no longer responsive to low-dose ephrin-A1 treatment (Fig. $4 a, b, d$; supplemental Fig. $2 B$, available at www.jneurosci.org as supplemental material). Therefore, FAK appears to be necessary for the inhibitory effects of Eprhin-A1 on neurite outgrowth.

To determine whether FAK regulates point contact dynamics downstream of ephrin-A1, paxillin-GFP was coexpressed with myc-tagged FRNK or combined with

FAKmo in neurons. FAK-deficient growth cones were imaged 10 min before and after the addition of $1 \mu \mathrm{g} \mathrm{ml}^{-1}$ ephrin-Al, as described previously. Interestingly, point contacts of FAKdeficient neurons were more stable compared with control growth cones even before ephrin-A1 treatment (supplemental Movie 2, supplemental Fig. 2C, available at www.jneurosci.org as supplemental material). The average lifetime of point contacts in FAK-deficient growth cones was $55 \pm 7.8 \%$ longer compared with point contacts of wild-type growth cones. This is not an unexpected result given that FAK has been implicated in both the assembly and turnover of focal adhesions (Webb et al., 2004), suggesting optimal adhesion dynamics requires balanced or dynamic FAK activity. However, addition of ephrin-A1 to FAKdeficient growth cones does not result in any further change in point contacts dynamics. Neither point contact lifetime (Fig. 4e) nor frequency of formation (Fig. 4f; supplemental Fig. $2 D$, available at www.jneurosci.org as supplemental material) significantly changes in response to ephrin-A1 in both FRNK-expressing and FAKmo-containing growth cones. Importantly, the effects of ephrin treatment on growth cone motility and point contact dynamics can be rescued in FAK knockdown neurons by overexpressing exogenous chick GFP-FAK (Fig. $4 c-f$ ), suggesting the effects of the FAK morpholino are specific. Together, these data suggest that activation of FAK is required for growth cones to respond ephrin-A1.

As our evidence suggests that Src acts as an upstream modulator of FAK activity (Fig. 3), we tested whether directly inhibiting Src with PP2 also prevented the effects of ephrin-A1 on growth cone motility and point contact dynamics. Consistent with reduced adhesion dynamics observed in FAK-deficient neurons (supplemental Fig. 2, available at www.jneurosci.org as supplemental material), we find that acute treatment with PP2 results in

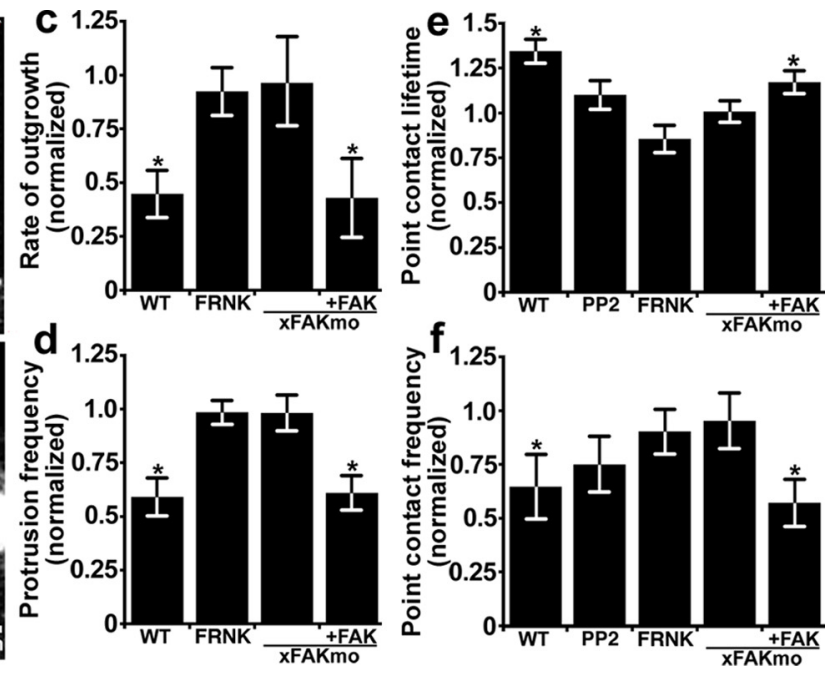

Figure 4. FAK and Src are required for ephrin-A1-induced changes in growth cone motility and point contact dynamics. $\boldsymbol{a}, A$ cone expressing GFP-FRNK was imaged at 10 s intervals for $10 \mathrm{~min}$ before and after the addition of $1 \mu \mathrm{g} \mathrm{ml}^{-1}$ ephrin-A1.

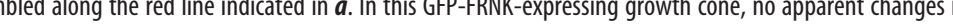
expressing paxillin-GFP together with myc-FRNK or FAKmo ( \pm chick GFP-FAK) normalized to each pretreatment lifetime and 列 10 min before ephrin-A1 addition. There is no significant change in the average point contact lifetime $(\boldsymbol{e})$ or ${ }^{*} p<0.05$ (paired Student's $t$ test used in $\boldsymbol{c}, \boldsymbol{d}, \boldsymbol{f}$ ). WT, Wild type. Scale bars, $5 \mu \mathrm{m}$ or as indicated.

an immediate stabilization of existing growth cone adhesions and a decrease in new adhesion formation (supplemental Fig. 3, available at www.jneurosci.org as supplemental material). These changes are associated with a near-complete cessation of protrusion and growth cone stalling (data not shown). Importantly, growth cones stabilized by Src inhibition no longer respond to ephrin-A1 with any further significant changes in point contact assembly or turnover (supplemental Fig. 3, available at www. jneurosci.org as supplemental material). The robust effects of pharmacological inhibition of Src on growth cone motility and point contact dynamics suggest that PP2 treatment may provide a more broad or complete inhibition compared with molecular inhibitors of FAK function.

Ephrin-A1 increases pY397-FAK differentially in nasal versus temporal RGC growth cones

EphA receptors are expressed in an increasing gradient along the nasal-temporal axis of the retina of all species examined (Cheng et al., 1995; Feldheim et al., 2000; Nakagawa et al., 2000; Gosse et al., 2008). Moreover, many studies have shown that temporal axons are more sensitive than nasal axons to the growth inhibitory effects of A-type ephrins (Cox et al., 1990; Roskies and O’Leary, 1994; Brennan et al., 1997; Weinl et al., 2005). Since we find that FAK phosphorylation occurs downstream of ephrin-A1, it is expected that activation of FAK by ephrin-A1 may occur differentially in nasal versus temporal axons. To determine whether nasal and temporal RGC axons are differentially sensitive to low doses of ephrin-A1, extreme nasal or temporal regions of eye primordia were cultured as explants on LN (Fig. 5a) (see Materials and Methods). The rate of axon outgrowth by nasal and temporal RGC axons was measured before and after addition of 1 $\mu \mathrm{g} \mathrm{ml}^{-1}$ ephrin-A1. Interestingly, before ephrin-A1 addition, the rate of temporal axon outgrowth was significantly faster than 


\section{a}
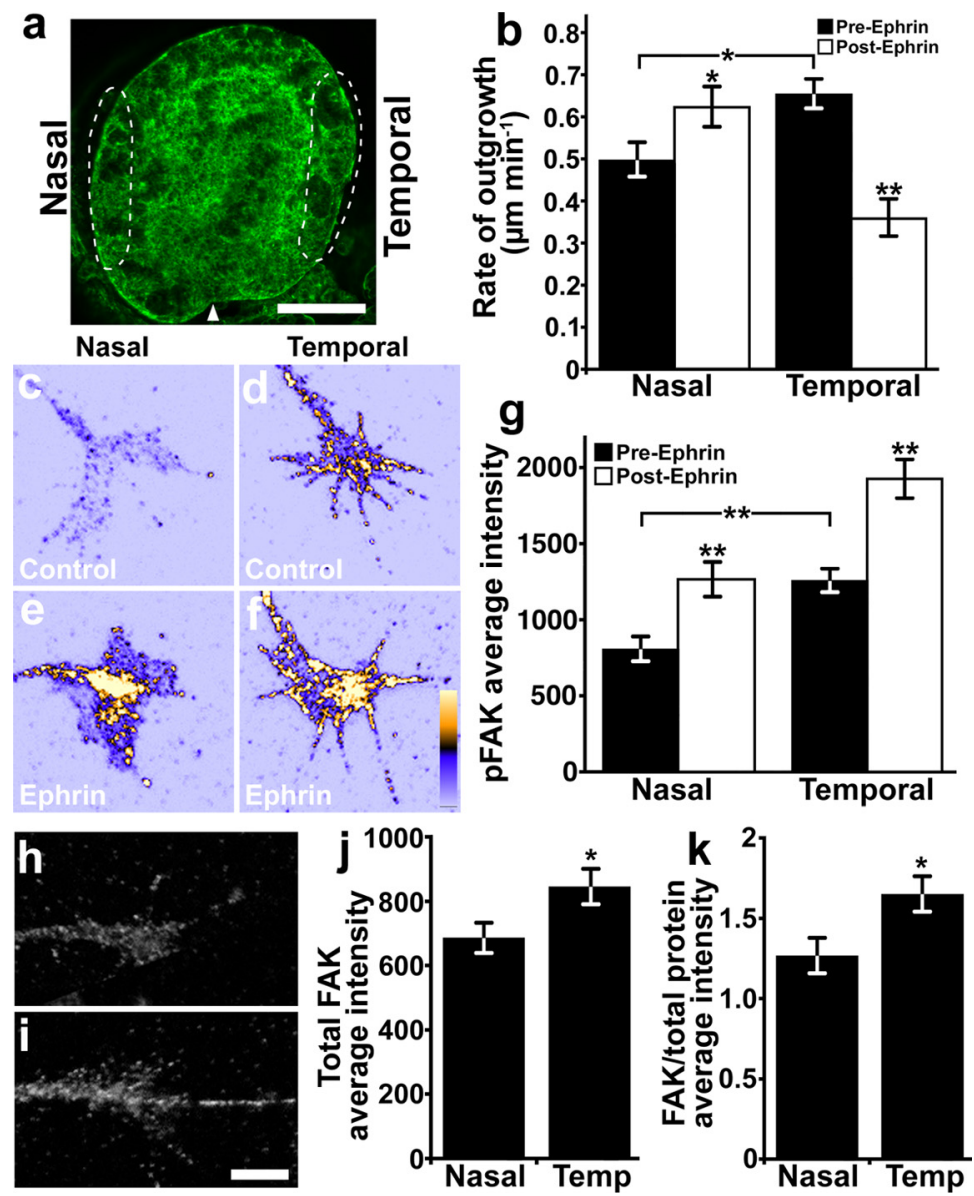

Figure 5. Nasal and temporal retinal neurons exhibit opposite responses to ephrin and differential FAK expression levels. a, Projected confocal z-series through a whole-mount stage 29 Xenopus eye labeled with A488-phalloidin. Extreme nasal or temporal $1 / 8$ to $1 / 10$ of eye primordia (dashed regions) were isolated and cultured on LN for $18-24 \mathrm{~h}$. Arrowhead indicates the ventral fissure. $\boldsymbol{b}$, The average rates of neurite outgrowth by nasal and temporal axons over $15 \mathrm{~min}$ periods before and after the addition of $1 \mu \mathrm{g} \mathrm{ml}^{-1}$ ephrin-A1. Ephrin-A1 promotes nasal axon outgrowth, while the same treatment significantly decreased the rate of temporal axon outgrowth. $n \geq 54$ neurons from four cultures for each condition. $\mathbf{c}-\boldsymbol{f}$, Representative RGC growth cones labeled for pY-397 FAK and pseudocolored based on fluorescence intensity (warmer colors indicate greater intensity). Growth cones from nasal and temporal RGC axons were treated for 5 min with control medium $(\boldsymbol{c}, \boldsymbol{d})$ or $2 \mu \mathrm{g} \mathrm{ml}^{-1}$ ephrin-A1 $(\boldsymbol{e}, \boldsymbol{f})$ before immunolabeling. Note that even without ephrin-A1 treatment, temporal growth cones $(\boldsymbol{d})$ appear to have more pY-397 FAK labeling than nasal growth cones (c), while both increase pY-397 FAK after ephrin treatment. $\boldsymbol{g}$, The average fluorescence intensities of pY-397 FAK immunolabeling measured within growth cones. Before ephrin stimulation, temporal growth cones have significantly higher baseline PY-397 FAK levels than nasal growth cones, but ephrin-A1 significantly increases pY-397 FAK in both nasal and temporal growth cones over control. $n \geq 69$ growth cones for each condition. $\boldsymbol{h}, \boldsymbol{i}$, Representative RGC growth cones isolated from nasal $(\boldsymbol{h})$ and temporal $(\boldsymbol{i})$ retina and labeled for total FAK. $\boldsymbol{j}$, The average fluorescence intensities of total FAK immunolabeling measured within growth cones from nasal and temporal retina. $\boldsymbol{k}$, The average fluorescence intensities of total FAK immunolabeling normalized to total protein shows that significantly more FAK is expressed in temporal compared with nasal RGC growth cones. ${ }^{*} p<0.05 .{ }^{* *} p<0.001$ (paired Student's $t$ test in $\boldsymbol{b}$ ). Scale bars: $\boldsymbol{a}, 100 \mu \mathrm{m} ; \boldsymbol{c}-\boldsymbol{f}, \boldsymbol{h}, \boldsymbol{i}, 5 \mu \mathrm{m}$.

nasal axon outgrowth (Fig. 5b). However, upon the addition of 1 $\mu \mathrm{g} \mathrm{ml}{ }^{-1}$ ephrin-A1, these baseline differences reversed, as temporal axons significantly slowed, while nasal axons significantly accelerated.

To determine whether the levels of p-FAK are differentially regulated in nasal versus temporal axons in response to ephrinA1, neurons from extreme nasal or temporal regions of eye primordia were treated for $5 \mathrm{~min}$ with $2 \mu \mathrm{g} \mathrm{ml}{ }^{-1}$ ephrin-A1 or control media and then fixed and immunostained using the $\mathrm{pY}$ 397 FAK antibody (Fig. 5c-f). The level of pY-397 FAK in growth cones was quantified as described previously. Treatment with 2 $\mu \mathrm{g} \mathrm{ml}^{-1}$ ephrin-A1 significantly increased pY-397 FAK levels in both nasal and temporal growth cones, but pY-397 FAK was elevated to a higher absolute level in temporal growth cones com- pared with nasal growth cones (Fig. 5g; supplemental Fig. $4 A$, available at www. jneurosci.org as supplemental material). However, even before the addition of ephrin-A1, the levels of pY-397 FAK within temporal growth cones appeared significantly greater than in nasal growth cones (Fig. $5 c, d, g$ ). This difference could either be due to greater total FAK protein levels in temporal versus nasal neurons, or a higher proportion of active FAK that is independent of ephrin stimulation. To differentiate between these two possibilities, we measured total FAK protein in nasal and temporal neurons by ICC and Western blot. We found by ICC that total FAK protein levels were also significantly higher in temporal relative to nasal axon growth cones cultured on LN (Fig. $5 h-k$ ). Immunoblotting for total FAK from lysates of nasal or temporal halves of stage 28 eye primordia also revealed a modest but not a significant enrichment of FAK protein in temporal versus nasal retina (supplemental Fig. 4B, available at www. jneurosci.org as supplemental material) ( $p=0.4597$, three independent trials, 20 retinas per sample). Modest temporal enrichment of FAK by Western blot relative to ICC may be due either to the specific enrichment of FAK within growth cones or specific enrichment within RGCs, since retinal lysates contain cell bodies from all retinal cell types. To determine whether differential FAK expression is specific to RGCs, we performed immunohistochemistry for FAK on horizontal cryostat sections over several developmental stages. We found that within the RGC layer of the retina, as defined by inner Islet-1 nuclear staining, there exists a nasal-to-temporal increasing gradient of FAK expression that persists over all developmental stages examined (Fig. 6). The FAK gradient is most clearly evident when FAK labeling is normalized to Islet-1 staining across the retina (supplemental Fig. 5, available at www.jneurosci.org as supplemental material). Together, these data suggest that temporal RGC axons are more sensitive to ephrin-A1 than nasal RGC axons and that higher sensitivity correlates with elevated levels of both total and pY-397FAK.

FAK is required for correct targeting of RGC axons in vivo A-type ephrins and their receptors are known for their role in topographic mapping of RGC axons onto the optic tectum (Lemke and Reber, 2005). Ephrin-A ligands are distributed in an increasing gradient along the anterior-to-posterior (A-P) axis of the optic tectum. Nasal RGC axons are less sensitive to ephrin-As due to low EphA receptor expression and project to areas of relatively high ephrin-A concentration in the posterior tectum (Carvalho et al., 2006). On the other hand, temporal RGC axons are more sensitive to ephrin-A due to high EphA expression and 
remain in the anterior tectum (Holt and Harris, 1983; Sakaguchi and Murphey, 1985; Mann et al., 2004). Our results indicate that FAK protein expression and phosphorylation levels also increase across the nasal-to-temporal axis in the retina, suggesting that FAK activity may function in the differential targeting of retinal axons onto the tectum in vivo.

To determine whether FAK is required for the correct positioning of RGC axons along the A-P axis within the optic tectum, we reduced FAK activity in two ways. First, GFP or GFP-FRNK mRNA was introduced into a single identified dorsal blastomere of eight-cell-stage Xenopus embryos, targeting expression to the right side of the head (Hirose and Jacobson, 1979). Importantly, this approach assures that the contralateral tectum, in which labeled axons project, is composed primarily of wild-type tissue (supplemental Fig. $6 A, B$, available at www.jneurosci.org as supplemental material). However, while we were able to label relatively robust retinotectal axon projections, suggesting that FRNK expression did not grossly interfere with RGC survival or axon extension, the retinal morphology of GFP-FRNKexpressing embryos was clearly disrupted (supplemental Fig. $7 A-I$, available at www.jneurosci.org as supplemental material), consistent with reports showing FAK to be important for cell proliferation and survival (Mitra et al., 2005). Therefore, we also expressed of FRNK under control of a heat shock promoter $(h s p)$ to delay expression until after eye morphogenesis had begun. A transgene consisting of the zebrafish $h s p 70$ promoter driving expression of GFP-FRNK ( $h s p$ : GFP-FRNK) was introduced at the one- or two-cell stage (Ogino et al., 2006), and embryos were heat shocked at stage 28 , when RGCs are first born (supplemental Fig. 8, available at www. jneurosci.org as supplemental material). The layering of Islet-1positive cells in $h s p$ :GFP-FRNK eyes appeared grossly similar to the wild type (supplemental Fig. $7 J-R$, available at www. jneurosci.org as supplemental material).

For all experiments, labeled embryos were fixed at stages 45 47, when RGC axons have begun to arborize within the tectum and segregation of nasal and temporal axons is readily apparent (Sakaguchi and Murphey, 1985). Nasal and temporal RGC axon projections were visualized by injecting two different fluorescent lipophilic tracers into opposite sides of GFP-positive eyes targeting nasal (DiD) and temporal (DiI) retina (supplemental Fig. $6 C, D$, available at www.jneurosci.org as supplemental material). Analysis of confocal $z$-stacks through the optic tecta from control (GFP) and dominant-negative FAK (GFP-FRNK and $h s p$ :GFPFRNK)-expressing embryos shows that suppression of FAK function leads to RGC targeting errors (Fig. 7). In control embryos, DiI-labeled temporal axons typically terminated anterior to DiDlabeled nasal axons (Fig. $7 a-d$ ). To assure accurate comparison of mapping, we measured the relative positions of temporal versus nasal RGC axon arbors along the A-P axis by two independent methods. The first measurement was the degree of overlap by the Pearson's correlation coefficient between the DiI- and DiD- labeled axons (Fig. 7m) (see Materials and Methods). In the second measurement, we calculated the CMs of DiI- and DiD-labeled RGC axon arborizations and measured the distance between the temporal and nasal arborizations for control and FRNK neurons (Fig. 7n). By both measurements, DiI-labeled temporal axons only partially overlapped with DiD-labeled nasal axons in control embryos (Fig. $7 d, m, n$ ). In contrast, in embryos expressing GFPFRNK or $h s p$ :GFP-FRNK, the position of axonal arborizations along the A-P axis was shifted (Fig. $7 e-n$ ). Unlike nasal and temporal axons of control embryos, the DiI and DiD labeling of GFP-FRNK RGCs overlapped extensively within the same area of the tectum (Fig. $7 h, l$ ). Importantly, the reduced separation between nasal and temporal arborizations in GFP-FRNK neurons was due in large part to a posterior shift of DiI-labeled temporal axons (supplemental Fig. 7, available at www.jneurosci.org as supplemental material), suggesting these neurons lost sensitivity to posterior repellants.

To test whether FAK regulates retinotectal mapping in another species and to use a more genetically tractable system, we examined the effects of FRNK expression on the formation of retinotectal projections in zebrafish. We obtained transgenic zebrafish embryos expressing Gal4 under control of the pou 433 promoter [ $T g(\text { pou4f3:Gal4 })^{\text {s311t }}$, which marks a subset of RGCs across the nasal-temporal axis of the eye (Xiao and Baier, 2007). These embryos were injected with DNA constructs containing either UAS:GFP or UAS:GFP-FRNK, resulting in mosaic expression. Embryos were fixed at 5 days postfertilization, and the positions of GFP-positive arbors within the tectum were analyzed by acquiring confocal $z$-stacks. Because relatively few neurons are labeled by pou 43 promoter-driven expression, we measured the 

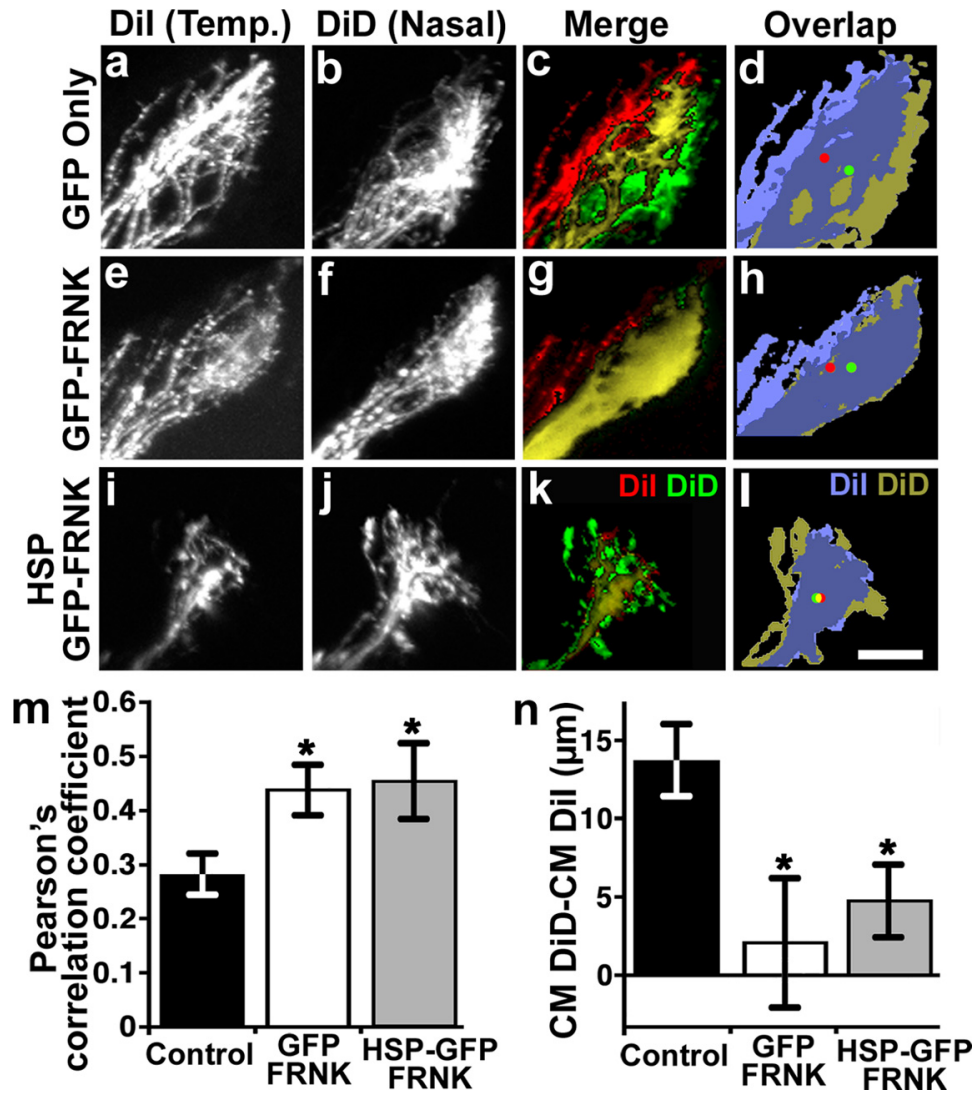

Figure 7. Inhibition of FAK activity disrupts anterior-posterior mapping of retinal axons in the optic tectum. $\boldsymbol{a}-\boldsymbol{I}$, Projected confocal z-stacks of lateral views of the optic tectum. Anterior is to the left. RGC axons from the contralateral eye express either GFP $(\boldsymbol{a}-\boldsymbol{d})$, GFP-FRNK $(\boldsymbol{e}-\boldsymbol{h})$, or $h$ sp:GFP-FRNK $(\boldsymbol{i}-\boldsymbol{l})$. In each embryo, temporal (Temp.) RGC axons were labeled with Dil $(\boldsymbol{a}, \boldsymbol{e}, \boldsymbol{i})$ and nasal axons were labeled with DiD $(\boldsymbol{b}, \boldsymbol{f}, \boldsymbol{j})$. Merged images of projected $z$-stacks show labeled temporal (red), nasal (green), and merged (yellow) axon arbors $(\boldsymbol{c}, \boldsymbol{g}, \boldsymbol{k})$. Merged images of thresholded projections of labeled temporal (light blue) and nasal (gold) axon arbors were used to determine each arbor CM indicated by red (temporal) and green (nasal) dots $(\boldsymbol{d}, \boldsymbol{h}, \boldsymbol{I})$. In control GFP-expressing embryos, nasal axons terminate posterior to temporal axons and exhibit only partial overlap $(\boldsymbol{c}, \boldsymbol{d})$. However, in embryos expressing GFP-FRNK or hsp:GFP-FRNK, nasal and temporal axons overlap extensively. $\boldsymbol{m}$, The Pearson's correlation coefficient was used to assess the degree of colocalization between nasal and temporal axons. $\boldsymbol{n}$, The difference between the Dil and DiD CM distances is significantly smaller in GFP-FRNK and $h s p$ :GFP-FRNK embryos, suggesting that nasal and temporal axons are no longer topographically segregated. $n \geq 8$ embryos for each condition. ${ }^{*} p<0.05$. Scale bar, $30 \mu \mathrm{m}$.
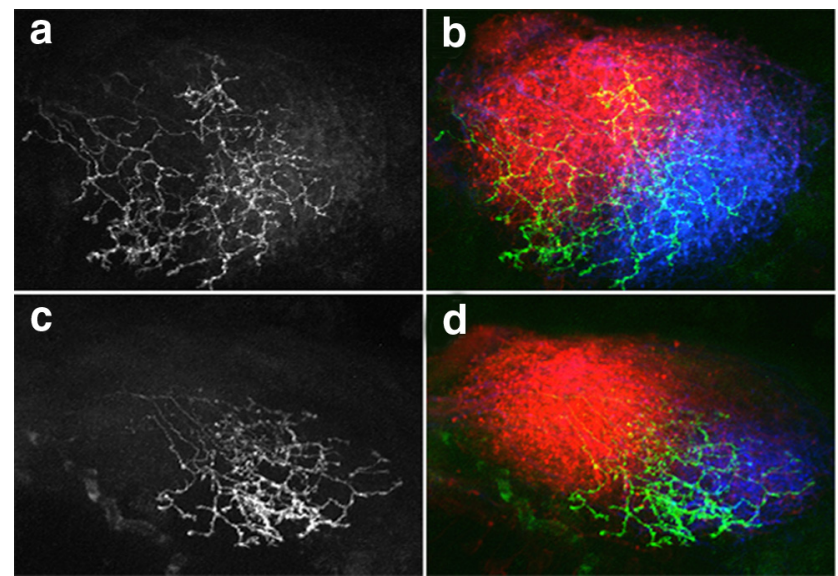

Figure 8. Inhibition of FAK activity disrupts anterior-posterior mapping of retinal axons in the zebrafish optic tectum. $\boldsymbol{a}-\boldsymbol{d}$, Projected confocal $z$-stacks of dorsal views of the optic tectum of a zebrafish embryo at 5 days postfertilization. Anterior is to the upper left. RGC axons from the contralateral eye express either GFP $(\boldsymbol{a}, \boldsymbol{b})$ or GFP-FRNK $(\boldsymbol{c}, \boldsymbol{d})$ under control of the pou4f3 promoter. Merged images of projected z-stacks show temporal RGC axons labeled with Dil (red), nasal axons labeled with DiD (blue), and GFP-expressing (green) axon arbors. Control GFP-expressing RGCs arborize widely across the tectum $(\boldsymbol{a}, \boldsymbol{b})$, while GFP-FRNK-expressing RGCs arborizemore posteriorly $(\boldsymbol{c}, \boldsymbol{d})$. Scalebar, $30 \mu \mathrm{m}$. arbor position of only the GFP- and GFPFRNK-positive axons across the predominantly wild-type RGC axons labeled by $\mathrm{DiD}$ and DiI injections, as described previously. Similar to the effects we observed in Xenopus, we find that GFP-FRNKexpressing RGC arbors are shifted toward the posterior tectum (Fig. $8 c, d$ ), while the average arbor position of control GFPexpressing RGCs showed no anteriorposterior bias (Fig. 8a,b). RGC arbor position along the A-P axis of the tectum was determined by measuring the center of mass of the thresholded GFP fluorescence signal. The average position of arbors, expressed as a percentage of the total length of the tectum, shows that GFPFRNK-expressing neurons are shifted posteriorly relative to control GFP arbors $(57 \% \pm 2.0 \%$ vs $49 \pm 3.0 \%, n \geq 8$ embryos for each condition, $p<0.05)$. Together, these data suggest that reducing FAK activity disrupts the normal positioning of RGC axons along the A-P axis of the optic tectum and that FAK is required for axons to interpret repellant cues expressed along this axis, such as a gradient of ephrin-A ligands.

\section{Discussion}

Eph and integrin receptors activate common downstream signaling pathways, yet the effects of ephrin on integrin-dependent adhesion of growth cones remain unclear. Here we report that a subcollapsing dose of ephrin-A1 stabilizes adhesive point contacts and reduces growth cone motility on integrin-dependent substrata. The effects of ephrin-A1 depend on activation of the adhesion-associated kinase FAK, which is phosphorylated and dephosphorylated at distinct residues in response to ephrin-A1. Similar results were observed when growth cones were treated with ephrin-A5 (supplemental Fig. 10, available at www.jneurosci. org as supplemental material), suggesting that this may be a general mechanism of ephrin-A signaling. We also find that FAK protein levels are expressed in an increasing nasal-to-temporal gradient within the retina similar to EphA receptors, and that temporal retinal axons are inhibited by ephrin-A due in part to higher FAK expression. Interestingly, low relative FAK expression in nasal compared with temporal RGC growth cones is associated with a slower baseline rate of neurite outgrowth. Moreover, the growth stimulating effects of ephrin-A1 on nasal axons suggests that a moderate level of active FAK is optimal for neurite outgrowth. Finally, we show that FAK activity is required for proper retinotopic mapping of axons along the anterior-posterior axis of the Xenopus and zebrafish optic tectum, which is mediated primarily by A-type ephrins. Together, our results suggest that ephrins control retinotopic mapping by differential activation of FAK, which controls growth cone motility by regulating integrin-based adhesion site dynamics.

Cell migration is controlled by coordination between the formation of adhesions at the leading edge and the disassembly of 
adhesions rearward (Kaverina et al., 2002). Therefore, extracellular factors could regulate cell motility by altering either the rate of assembly or turnover of adhesion sites. We find that ephrin-A1 reduces new point contact formation, but stabilizes existing point contacts, resulting in a significant slowing or pausing of growth cone migration. Importantly, stabilization of paxillin-containing adhesions by ephrin-A1 was observed only at a subcollapsing dose of ephrin-A1. These results suggest that although loss of adhesion may be necessary for full growth cone collapse or local collapse during turning, increased adhesion lifetime may function to pause or stop axon extension at low ephrin concentrations. In addition, we found that FAK is at least partially required for full growth cone collapse at higher concentrations of ephrin-A1 (supplemental Fig. 11, available at www.jneurosci.org as supplemental material), whereas other studies have suggested that ephrin-induced growth cone collapse is due to RhoA overactivation via ephexin (Wahl et al., 2000; Shamah et al., 2001). Therefore, growth cone collapse at high doses of ephrin-A versus point contact stabilization at low doses may depend on the extent of FAK phosphorylation and RhoA activation.

We found that nasal RGCs express lower endogenous FAK than temporal RGCs and have a lower basal rate of axon outgrowth on LN before ephrin treatment (Fig. 5). However, stimulation by ephrin increases the extension rate of nasal axons but strongly inhibits the extension of temporal axons. Bifunctional effects of ephrin on RGC axon extension have been described previously in other systems (Hansen et al., 2004). Interestingly, stimulation of nasal axon outgrowth by ephrin is associated with the elevation of pY-397 FAK in nasal axons to levels comparable to temporal axons before treatment with ephrin (Fig. 5b,g). On the other hand, temporal axons, which have moderate baseline pY-397 FAK and fast outgrowth before ephrin treatment, slow dramatically and exhibit very high pY-397 FAK in response to ephrin. These results suggest that moderate levels of active FAK promote maximum outgrowth and that changes above or below this optimal set-point inhibit outgrowth. While our results suggest that reduced temporal axon outgrowth through FAK hyperactivation is due to the stabilization of point contacts, it is not clear whether stimulation of nasal axon outgrowth by ephrin-A1 also occurs through modulation of point contact dynamics. Modulation Y397-FAK phosphorylation and adhesion site dynamics may explain how chemoattractants like Netrin and BDNF also require FAK function (Li et al., 2004; Ren et al., 2004).

Several lines of evidence suggest that FAK functions downstream of ephrin-A in retinotopic mapping. First, FAK is expressed in a low-to-high, nasal-to-temporal gradient across the ganglion cell layer within the retina, similar to EphA receptors. Second, in response to ephrin-A1, temporal growth cones increased their absolute levels of tyrosine-phosphorylated FAK (pY-FAK) to a greater extent than nasal growth cones. Finally, inhibiting FAK function in both Xenopus and zebrafish RGCs disrupts retinotopic mapping similar to ephrin-A and EphA loss of function. For example, a posterior shift of temporal axon termination zones within the superior colliculus was observed in ephrin-A2/ephrin-A5 knock-out mice (Feldheim et al., 2000), as well as in mice expressing dominant-negative EphA receptors (Feldheim et al., 2004). Although the termination zones in the Xenopus and zebrafish tecta are much broader relative to the chick tectum and mouse colliculus (Lemke and Reber, 2005), the magnitude of the nasal and temporal map shifts we observe (supplemental Fig. 9, available at www.jneurosci.org as supplemental material) are comparable to dorsal-ventral shifts ob- served when ephrinB/EphB signaling is inhibited in Xenopus (Mann et al., 2004).

Studies in Xenopus have shown that nasal axon arbors undergo more dynamic branch remodeling while temporal branch patterns remain relatively stable (O'Rourke and Fraser, 1990). Interestingly, FAK has recently been shown to inhibit branch formation of both Purkinje and hippocampal neurons (Rico et al., 2004). Together with our results, these studies suggest that higher FAK expression in temporal axons may function to inhibit axon branch formation in response to increased ephrin-A in the posterior tectum. Interestingly, in chick and mouse retinotopic mapping, temporal axons initially overshoot their proper termination site, but ultimately undergo interstitial branching at the appropriate anterior-posterior position, followed by retraction of the posterior processes (Yates et al., 2001; Rashid et al., 2005). While the initial targeting of nasal and temporal axons in Xenopus is more precise, the tectum itself is still growing during this time (Straznicky and Gaze, 1972), and FAK may limit branching of temporal axons into newly expanded posterior tectal space. It will be interesting to determine whether nasal versus temporal differences in FAK expression also occur in chick and mouse retina, in which branching plays a larger role in retinotopic mapping.

\section{References}

Bourgin C, Murai KK, Richter M, Pasquale EB (2007) The EphA4 receptor regulates dendritic spine remodeling by affecting betal-integrin signaling pathways. J Cell Biol 178:1295-1307.

Brennan C, Monschau B, Lindberg R, Guthrie B, Drescher U, Bonhoeffer F, Holder N (1997) Two Eph receptor tyrosine kinase ligands control axon growth and may be involved in the creation of the retinotectal map in the zebrafish. Development 124:655-664.

Carter N, Nakamoto T, Hirai H, Hunter T (2002) EphrinAl-induced cytoskeletal re-organization requires FAK and p130(cas). Nat Cell Biol 4:565-573.

Carvalho RF, Beutler M, Marler KJ, Knöll B, Becker-Barroso E, Heintzmann $\mathrm{R}, \mathrm{Ng} \mathrm{T}$, Drescher U (2006) Silencing of EphA3 through a cis interaction with ephrinA5. Nat Neurosci 9:322-330.

Cheng HJ, Nakamoto M, Bergemann AD, Flanagan JG (1995) Complementary gradients in expression and binding of ELF-1 and Mek4 in development of the topographic retinotectal projection map. Cell 82:371-381.

Cox EC, Müller B, Bonhoeffer F (1990) Axonal guidance in the chick visual system: posterior tectal membranes induce collapse of growth cones from the temporal retina. Neuron 4:31-37.

Feldheim DA, Kim YI, Bergemann AD, Frisén J, Barbacid M, Flanagan JG (2000) Genetic analysis of ephrin-A2 and ephrin-A5 shows their requirement in multiple aspects of retinocollicular mapping. Neuron 25:563-574.

Feldheim DA, Nakamoto M, Osterfield M, Gale NW, DeChiara TM, Rohatgi R, Yancopoulos GD, Flanagan JG (2004) Loss-of-function analysis of EphA receptors in retinotectal mapping. J Neurosci 24:2542-2550.

Frame MC, Fincham VJ, Carragher NO, Wyke JA (2002) v-Src's hold over actin and cell adhesions. Nat Rev Mol Cell Biol 3:233-245.

Geiger B, Bershadsky A, Pankov R, Yamada KM (2001) Transmembrane crosstalk between the extracellular matrix-cytoskeleton crosstalk. Nat Rev Mol Cell Biol 2:793-805.

Gomez TM, Roche FK, Letourneau PC (1996) Chick sensory neuronal growth cones distinguish fibronectin from laminin by making substratum contacts that resemble focal contacts. J Neurobiol 29:18-34.

Gómez TM, Harrigan D, Henley J, Robles E (2003) Working with Xenopus spinal neurons in live cell culture. Methods Cell Biol 71:129-156.

Gosse NJ, Nevin LM, Baier H (2008) Retinotopic order in the absence of axon competition. Nature 452:892-895.

Halloran MC, Sato-Maeda M, Warren JT, Su F, Lele Z, Krone PH, Kuwada JY, Shoji W (2000) Laser-induced gene expression in specific cells of transgenic zebrafish. Development 127:1953-1960.

Hansen MJ, Dallal GE, Flanagan JG (2004) Retinal axon response to ephrin-as shows a graded, concentration-dependent transition from growth promotion to inhibition. Neuron 42:717-730.

Hirose G, Jacobson M (1979) Clonal organization of the central nervous 
system of the frog. I. Clones stemming from individual blastomeres of the 16-cell and earlier stages. Dev Biol 71:191-202.

Holt CE, Harris WA (1983) Order in the initial retinotectal map in Xenopus: a new technique for labelling growing nerve fibres. Nature 301:150-152.

Hynes RO (2002) Integrins: bidirectional, allosteric signaling machines. Cell 110:673-687.

Jeon J, Lee H, Park H, Lee JH, Choi S, Hwang J, Han IO, Oh ES (2007) Phosphorylation of focal adhesion kinase at Tyrosine 407 negatively regulates Ras transformation of fibroblasts. Biochem Biophys Res Commun 364:1062-1066.

Jurney WM, Gallo G, Letourneau PC, McLoon SC (2002) Racl-mediated endocytosis during ephrin-A2- and semaphorin $3 \mathrm{~A}$-induced growth cone collapse. J Neurosci 22:6019-6028.

Kaverina I, Krylyshkina O, Small JV (2002) Regulation of substrate adhesion dynamics during cell motility. Int J Biochem Cell Biol 34:746-761.

Kirchner J, Kam Z, Tzur G, Bershadsky AD, Geiger B (2003) Live-cell monitoring of tyrosine phosphorylation in focal adhesions following microtubule disruption. J Cell Sci 116:975-986.

Knöll B, Drescher U (2004) Src family kinases are involved in EphA receptor-mediated retinal axon guidance. J Neurosci 24:6248-6257.

Lemke G, Reber M (2005) Retinotectal mapping: new insights from molecular genetics. Annu Rev Cell Dev Biol 21:551-580.

Li W, Lee J, Vikis HG, Lee SH, Liu G, Aurandt J, Shen TL, Fearon ER, Guan JL, Han M, Rao Y, Hong K, Guan KL (2004) Activation of FAK and Src are receptor-proximal events required for netrin signaling. Nat Neurosci 7:1213-1221.

Lim Y, Han I, Jeon J, Park H, Bahk YY, Oh ES (2004) Phosphorylation of focal adhesion kinase at tyrosine 861 is crucial for Ras transformation of fibroblasts. J Biol Chem 279:29060-29065.

Mann F, Harris WA, Holt CE (2004) New views on retinal axon development: a navigation guide. Int J Dev Biol 48:957-964.

Miao H, Burnett E, Kinch M, Simon E, Wang B (2000) Activation of EphA2 kinase suppresses integrin function and causes focal-adhesion-kinase dephosphorylation. Nat Cell Biol 2:62-69.

Mitra SK, Hanson DA, Schlaepfer DD (2005) Focal adhesion kinase: in command and control of cell motility. Nat Rev Mol Cell Biol 6:56-68.

Nakagawa S, Brennan C, Johnson KG, Shewan D, Harris WA, Holt CE (2000) Ephrin-B regulates the ipsilateral routing of retinal axons at the optic chiasm. Neuron 25:599-610.

Nieuwkoop PD, Faber J (1994) Normal table of Xenopus laevis (Daudin). New York: Garland.

Ogino H, McConnell WB, Grainger RM (2006) Highly efficient transgenesis in Xenopus tropicalis using I-SceI meganuclease. Mech Dev 123:103-113

O'Rourke NA, Fraser SE (1990) Dynamic changes in optic fiber terminal arbors lead to retinotopic map formation: an in vivo confocal microscopic study. Neuron 5:159-171.

Pasquale EB (2005) Eph receptor signalling casts a wide net on cell behaviour. Nat Rev Mol Cell Biol 6:462-475.

Rashid T, Upton AL, Blentic A, Ciossek T, Knöll B, Thompson ID, Drescher U (2005) Opposing gradients of ephrin-As and EphA7 in the superior colliculus are essential for topographic mapping in the mammalian visual system. Neuron 47:57-69.

Ren XR, Ming GL, Xie Y, Hong Y, Sun DM, Zhao ZQ, Feng Z, Wang Q, Shim S, Chen ZF, Song HJ, Mei L, Xiong WC (2004) Focal adhesion kinase in netrin-1 signaling. Nat Neurosci 7:1204-1212.
Renaudin A, Lehmann M, Girault J, McKerracher L (1999) Organization of point contacts in neuronal growth cones. J Neurosci Res 55:458-471.

Rico B, Beggs HE, Schahin-Reed D, Kimes N, Schmidt A, Reichardt LF (2004) Control of axonal branching and synapse formation by focal adhesion kinase. Nat Neurosci 7:1059-1069.

Robles E, Gomez TM (2006) Focal adhesion kinase signaling at sites of integrin-mediated adhesion controls axon pathfinding. Nat Neurosci 9:1274-1283.

Robles E, Huttenlocher A, Gomez TM (2003) Filopodial calcium transients regulate growth cone motility and guidance through local activation of calpain. Neuron 38:597-609.

Roskies AL, O'Leary DD (1994) Control of topographic retinal axon branching by inhibitory membrane-bound molecules. Science 265:799803.

Sahin M, Greer PL, Lin MZ, Poucher H, Eberhart J, Schmidt S, Wright TM, Shamah SM, O'connell S, Cowan CW, Hu L, Goldberg JL, Debant A, Corfas G, Krull CE, Greenberg ME (2005) Eph-dependent tyrosine phosphorylation of ephexin 1 modulates growth cone collapse. Neuron 46:191-204.

Sakaguchi DS, Murphey RK (1985) Map formation in the developing Xenopus retinotectal system: an examination of ganglion cell terminal arborizations. J Neurosci 5:3228-3245.

Schindelholz B, Reber BF (1999) Quantative estimation of F-actin in single growth cones. Methods 18:487-492.

Schlaepfer DD, Mitra SK, Ilic D (2004) Control of motile and invasive cell phenotypes by focal adhesion kinase. Biochim Biophys Acta 1692:77-102.

Shamah SM, Lin MZ, Goldberg JL, Estrach S, Sahin M, Hu L, Bazalakova M, Neve RL, Corfas G, Debant A, Greenberg ME (2001) EphA receptors regulate growth cone dynamics through the novel guanine nucleotide exchange factor ephexin. Cell 105:233-244.

Straznicky K, Gaze RM (1972) The development of the tectum in Xenopus laevis: an autoradiographic study. J Embryol Exp Morphol 28:87-115.

Thermes V, Grabher C, Ristoratore F, Bourrat F, Choulika A, Wittbrodt J, Joly JS (2002) I-SceI meganuclease mediates highly efficient transgenesis in fish. Mech Dev 118:91-98.

Turner CE (2000) Paxillin and focal adhesion signalling. Nat Cell Biol 2:E231-E236.

Varnum-Finney B, Reichardt LF (1994) Vinculin-deficient PC12 cell lines extend unstable lamellipodia and filopodia and have a reduced rate of neurite outgrowth. J Cell Biol 127:1071-1084.

Wahl S, Barth H, Ciossek T, Aktories K, Mueller BK (2000) Ephrin-A5 induces collapse of growth cones by activating Rho and Rho kinase. J Cell Biol 149:263-270.

Webb DJ, Donais K, Whitmore LA, Thomas SM, Turner CE, Parsons JT, Horwitz AF (2004) FAK-Src signalling through paxillin, ERK and MLCK regulates adhesion disassembly. Nat Cell Biol 6:154-161.

Weinl C, Becker N, Loeschinger J (2005) Responses of temporal retinal growth cones to ephrinA5-coated beads. J Neurobiol 62:219-230.

Woo S, Gomez TM (2006) Racl and RhoA promote neurite outgrowth through formation and stabilization of growth cone point contacts. J Neurosci 26:1418-1428.

Xiao T, Baier H (2007) Lamina-specific axonal projections in the zebrafish tectum require the type IV collagen Dragnet. Nat Neurosci 10:1529-1537.

Yates PA, Roskies AL, McLaughlin T, O'Leary DD (2001) Topographicspecific axon branching controlled by ephrin-As is the critical event in retinotectal map development. J Neurosci 21:8548-8563. 